Research Article

\title{
Analysis of Cholera Epidemic Controlling Using Mathematical Modeling
}

\author{
Kinfe Hailemariam Hntsa (iD) and Berhe Nerea Kahsay \\ Department of Mathematics, Aksum University, Axum, Ethiopia \\ Correspondence should be addressed to Kinfe Hailemariam Hntsa; kinfhail@gmail.com
}

Received 7 April 2020; Revised 25 May 2020; Accepted 3 June 2020; Published 17 August 2020

Academic Editor: Harvinder S. Sidhu

Copyright (c) 2020 Kinfe Hailemariam Hntsa and Berhe Nerea Kahsay. This is an open access article distributed under the Creative Commons Attribution License, which permits unrestricted use, distribution, and reproduction in any medium, provided the original work is properly cited.

\begin{abstract}
The purpose of this study is to see whether it is possible to eradicate the disease theoretically using mathematical modeling with the aid of numerical simulation when disease occurs in a population by implementing adequate preventive measures. For this, we consider a mathematical model for the transmission dynamics of cholera and its preventive measure as one cohort of individuals, namely, a protected cohort in addition to susceptible, infected, and recovered cohorts of individuals including the concentration of Vibrio cholerae in the contaminated aquatic reservoir with small modifications. We calculate the basic reproduction number, $\mathscr{R}_{0}$, and investigate the existence and stability of equilibria. The model possessed forward bifurcation. Moreover, we compute the sensitivity indices of each parameter in relating to $\mathscr{R}_{0}$ of the model. Numerical simulations are carried out to validate our theoretical results. The result indicates that the disease dies out in areas with adequate preventive measures and widespread and kills more people in areas with the inadequate preventive measures.
\end{abstract}

\section{Introduction}

The cholera epidemic is a fatal waterborne disease causing diarrhea, dehydration, and vomiting in an individual $[1,2]$. It is caused by a bacterium called Vibrio cholerae. Cholera is transmitted through ingesting contaminated drinks and food, contact with cholera patient's feces, and touching vomit and corpse killed by the bacterium without using protective agents [1-3]. The incubation period of cholera is less than 24 hours to 5 days. The infection is frequently asymptomatic. Not more than $25 \%$ of the infected persons become symptomatic; of these, $10-20 \%$ experience severe disease [1]. A continuous drastic loss of body fluids leads to dehydration, thus neglecting treatment as soon as the case occurs, and accelerates the death of the infected person within hours [4].

There are environmental factors that play a great role in the propagation of cholera infection. As Vibrio cholerae can move in the aquatic environment, every alteration in the hydrological cycle has a probability to affect the pathogenic concentration in water. The rain and its seasonal behavior, droughts and floods, can increase or decrease the transmission process [5].

Nevertheless, relevance has to be given to the environmental matrix in which the disease spreads into disease-free regions $[6,7]$ together with consideration on individual mobility and travelers carrying the disease in long-distance journeys. Susceptible people traveling on a daily basis may contact the disease in destination sites and take the disease back to the possibly uninfected communities where they regularly live. At the same time, infected individuals not showing severe symptoms can carry the illness releasing bacteria via their feces [8]. Finally, symptomatic infected individuals locally increase the bacteria concentration, which is, then, spread along the hydrological network.

Experiments for spreading of infectious disease in individual are unethical. Epidemiologists and other researchers use mathematical modeling and numerical simulation for scientific understanding about the dynamics and preventive method of an infectious disease, for determining sensitivities, changes of parameter values, and to forecasting [5, 9-19]. The models are based on cohorts, 
namely, susceptible, infected, and recovered, for individual population incorporated with some preventive measures such as treatment, vaccination, chlorination, hygienic, and sanitation through education. Treatment is more recommended when cases occur, that is, a short-time plan of controlling and eradicating the disease. Education is the most recommended option in the disease controlling and eradicating from the community whenever. Education is a long-term plan of controlling and eradicating the disease, especially in creating awareness of water treatment, sewage removal, food safety, personal hygiene, and environment sanitation [3, 20-22]. It is an undeniable truth that there are individual populations who have awareness on the adequate preventive measures of cholera diseases and compliance them. In our best knowledge, those populations are not considered as one cohort of individuals in addition to susceptible, infected, and recovered individual cohorts in order to analyse the dynamics, control, and eliminate cholera.

The objective of this study is to analyse qualitatively the controlling mechanisms of cholera epidemics in a population by implementation of adequate preventive measures.

\section{Formulation of Mathematical Modeling}

We divided the population denoted by $N(t)$ according to the infection status into $S(t)$ - susceptible, $I(t)$ - infected, $R(t)$ - recovered, and $U(t)$ - prevented individuals at given time $t$. Moreover, $B(t)$ is the amount of concentration of Vibro cholerae in an aquatic environment at time $t$. New susceptible individuals are recruited into the community at a rate of $b$ and $(1-p) \rho$, where $b$ is birth or immigration rate of individuals, $p$ is the fraction of the compliance of hygienic, ingestion of cholera bacterium and contact with cholera patients of susceptible and recovered cohort, and $\rho$ is the loss rate of immunity of recovered individuals. They either die from suffering at a rate $\mu$, where $\mu$ is the sum of the natural death rate and population-dependent death rate, or move to an infectious cohort by acquiring cholera through contact with the aquatic reservoir at a rate of (1p) $\beta(B /(B+K))$ or move to the protected cohort at a rate of $p \alpha$, where $\alpha$ is progression rate of susceptible individuals to the protected cohort due to the preventive method.

We note that an individual must consume at least the concentration, $K$, of Vibrios cholerae equivalent to an amount that increases the possibility of being infected to about $50 \%$ if they are to contact the infection. Infected individuals are assumed to recover at rate $\tau$. Infectious individuals who do not recover die at rate $\mu+d$, where $d$ is the cholera-induced death rate. Recovered individuals move to protected cohorts at a rate of $p \rho$. Infected individuals in the community shed the pathogen into the aquatic environment at rate $(1-\theta) \phi$, where $\theta$ is the fraction of the compliance of sanitation of an infected cohort and $\phi$ is the average contribution of each infected individual to the pathogen population of Vibrio cholerae. The individual population follows logistic growth due to intraspecific competition for limited environmental resources in a disease-free environment. The complete description of parameters is in Table 1.

We assumed that the adequate preventing measures are using of safe water for drinking, washing, and food preparation; disposing feces in a sanitary manner; removing or washing any bedding or clothing that might have contact with cholera-infected people; restraining a cholera-infected person from swimming even until two weeks after individuals recover; washing hands always with soap and clean water before preparing food, eating, feeding children, after using a latrine, and taking care of patients with diarrhea; cleaning food preparation areas; and storing treated water and food in clean and covered containers.

Based on these assumptions and the flow diagram in Figure 1, we formulate the following SIRUB model, which is a system of nonlinear autonomous ordinary differential equations:

$$
\begin{aligned}
& \frac{\mathrm{d} S}{\mathrm{~d} t}=b N+(1-p) \rho R-\left((1-p) \beta \frac{B}{B+K}+p \alpha+\mu\right) S, \\
& \frac{\mathrm{d} I}{\mathrm{~d} t}=(1-p) \beta \frac{B S}{B+K}-(\tau+d+\mu) I, \\
& \frac{\mathrm{d} R}{\mathrm{~d} t}=\tau I-(\rho+\mu) R, \\
& \frac{\mathrm{d} U}{\mathrm{~d} t}=p \alpha S+p \rho R-\mu U, \\
& \frac{\mathrm{d} B}{\mathrm{~d} t}=(1-\theta) \phi I+\left(b_{B}-\mu_{B}\right) B,
\end{aligned}
$$

where $\mu=\mu_{i}+\mu_{d} N$ and $N(t)=S(t)+I(t)+R(t)+U(t)$, and with initial conditions given, $S(0)=S_{0} \geq 0, I(0)=$ $I_{0} \geq 0, R(0)=R_{0} \geq 0, U(0)=U_{0} \geq 0, B(0)=B_{0} \geq 0$.

Theorem 1 (mathematically and epidemiologically well posed). Assuming that the initial conditions lie in $\Omega$, the dynamical system of equation (1) has a unique bounded positive solution in $\Omega$ for all time $t \geq 0$, where

$$
\Omega=\left\{(S, I, R, U, B) \in \mathbb{R}_{+}^{5}: S+I+R+U=N, 0 \leq N \leq L, 0 \leq B \leq \frac{(1-\theta) \phi L}{\left(\mu_{B}-b_{B}\right)}\right\},
$$


TABle 1: Parameters for system of (1), where $c$ is capita, $l$ is litter, and $T$ is time.

\begin{tabular}{|c|c|c|}
\hline Parameter & Parameter definition & Dimension \\
\hline$b$ & Constant recruitment (birth or immigration) rate of an individual & {$[b]=T^{-1}$} \\
\hline$\mu_{i}$ & Density-independent death rate of an individual & {$\left[\mu_{i}\right]=T^{-1}$} \\
\hline$\mu_{d}$ & Density-dependent death rate of an individual & {$\left[\mu_{d}\right]=c^{-1} T^{-1}$} \\
\hline$d$ & Disease-induced death rate of an individual & {$[d]=T^{-1}$} \\
\hline$\beta$ & Rates of ingesting Vibrio cholerae from the contaminated aquatic reservoir & {$[\beta]=T^{-1}$} \\
\hline$\tau$ & Progression rate of infected individuals to the recovered class due to treatment & {$[\tau]=T^{-1}$} \\
\hline$\alpha$ & Progression rate of susceptible individuals to the protected class due to the preventive method & {$[\alpha]=T^{-1}$} \\
\hline$\rho$ & The rate of loss of immunity of recovered individuals & {$[\rho]=T^{-1}$} \\
\hline$\phi$ & The average contribution of each infected individual to the pathogen population of Vibrio cholerae & {$[\phi]=l^{-1} T^{-1}$} \\
\hline$K$ & The concentration of Vibrio cholerae in food and water that yield $50 \%$ chance of acquiring cholera disease & {$[K]=c l^{-1}$} \\
\hline$p$ & $\begin{array}{c}\text { Fraction of the compliance of hygienic, ingestion of cholera bacterium and contact with cholera patients of the } \\
\text { susceptible and recovered cohort }\end{array}$ & {$[p]$} \\
\hline$\theta$ & Fraction of the compliance of sanitation of the infected cohort & {$[\theta]=1$} \\
\hline$b_{B}$ & Birth rate of Vibrio cholerae & {$\left[b_{B}\right]=T^{-1}$} \\
\hline$\mu_{B}$ & Death rate of Vibrio cholerae & {$\left[\mu_{B}\right]=T^{-1}$} \\
\hline
\end{tabular}

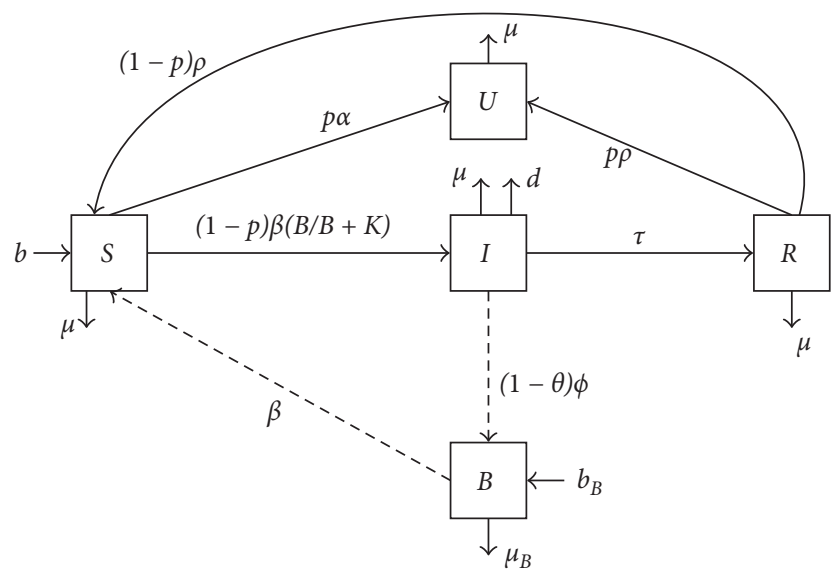

Figure 1: The schematic depiction of the dynamics and prevention measures of cholera.

provided that $\mu_{B}>b_{B}$.

Proof. We show first boundness. The total individual population becomes

$$
\begin{aligned}
\frac{\mathrm{d} N}{\mathrm{~d} t}=\frac{\mathrm{d} S}{\mathrm{~d} t}+\frac{\mathrm{d} I}{\mathrm{~d} t}+\frac{\mathrm{d} R}{\mathrm{~d} t}+\frac{\mathrm{d} U}{\mathrm{~d} t} & =b N-\mu N-\mathrm{d} I \\
& =N\left(b-\mu_{i}-\mu_{d} N\right)-\mathrm{d} I, \\
& =\left(b-\mu_{i}\right) N\left(1-\frac{N}{\left(b-\mu_{i}\right) / \mu_{d}}\right)-\mathrm{d} I \\
& =r_{h} N\left(1-\frac{N}{L}\right)-\mathrm{d} I,
\end{aligned}
$$

where $r_{h}=b-\mu_{i}$ and $L=\left(\left(b-\mu_{i}\right) / \mu_{d}\right)$ which are known as the intrinsic growth rate and carrying capacity of an individual population, respectively. In the absence of the disease $(I=0)$, equation (3) can be written as

$$
\frac{\mathrm{d} N}{\mathrm{~d} t} \leq r_{h} N\left(1-\frac{N}{L}\right) \Longrightarrow N(t) \leq \frac{L}{((L / N(0))-1) e^{-r_{h} t}+1} .
$$

Now, it is observed that

$$
0 \leq N(t) \leq \lim _{t \rightarrow \infty} \frac{L}{((L / N(0))-1) e^{-r_{h} t}+1}=L .
$$

Moreover, the last equation of the dynamical system can be expressed as

$$
\frac{\mathrm{d} B}{\mathrm{~d} t}+\left(\mu_{B}-b_{B}\right) B=(1-\theta) \phi I
$$


and its solution is given by

$$
B(t)=c e^{-\left(\mu_{B}-b_{B}\right) t}+\frac{(1-\theta) \phi I}{\left(\mu_{B}-b_{B}\right)} .
$$

Thus, we get

$$
\begin{aligned}
0 \leq B(t) & \leq \lim _{t \rightarrow \infty}\left(c e^{-\left(\mu_{B}-b_{B}\right) t}+\frac{(1-\theta) \phi I}{\left(\mu_{B}-b_{B}\right)}\right)=\frac{(1-\theta) \phi I}{\left(\mu_{B}-b_{B}\right)} \\
& \leq \frac{(1-\theta) \phi L}{\left(\mu_{B}-b_{B}\right)} .
\end{aligned}
$$

Therefore, $N(t)$ and $B(t)$ are bounded.

The dynamical system of equation (1) has a unique solution because the right-hand side of equation (1) is continuous with continuous partial derivatives in $\Omega$.

To show the positivity invariant, let us consider the first equation of system (1), and it becomes

$$
\frac{\mathrm{d} S}{\mathrm{~d} t} \geq-\left((1-p) \beta \frac{B}{B+K}+p \alpha+\mu\right) S .
$$

This gives

$$
S(t) \geq S_{0} e^{-((1-p) \beta(B /(B+K))+p \alpha+\mu) t} \geq 0 .
$$

Similarly, we get

$$
\begin{aligned}
& I(t) \geq I_{0} e^{-(\tau+d+\mu) t} \geq 0, \\
& R(t) \geq R_{0} e^{-(\rho+\mu) t} \geq 0, \\
& U(t) \geq U_{0} e^{-\mu t} \geq 0, \\
& B(t) \geq B_{0} e^{-\left(\mu_{B}-b_{B}\right) t} \geq 0 .
\end{aligned}
$$

The solutions in $\Omega$ are all nonnegative. Hence, the domain of biological significance is positively invariant and attracting. Therefore, the dynamical system in equation (1) has a unique bounded positive solution in $\Omega$.

\section{Stability Analysis of Equilibrium Points}

3.1. Disease-Free Equilibrium Point. The disease-free equilibrium point is the steady-state solutions determined when there is no disease $(I=0)$, and it is unique given by

$$
E_{0}=\left(S^{*}, 0,0, U^{*}, 0\right),
$$

where $S^{*}=(b /(p \alpha+b)) N^{*}$ and $U^{*}=(p \alpha /(p \alpha+b)) N^{*}$, $N^{*}=L$. It is observed that if $p \alpha \gg b$, then $(b /(p \alpha+b)) \approx 0$ and $(p \alpha /(p \alpha+b)) \approx 1$ which indicate that $S(t) \approx 0$ and $U(t) \approx N(t)$. From the biological point of view, the disease can be controlled and eliminated from the community when the rate of susceptible individuals moving to a protected cohort due to preventive methods is greater than the recruitment rate of individuals.

3.2. Basic Reproduction Number. The basic reproduction number represents the average number of new infected individuals that result from one infected individual introduced into a fully susceptible population. The basic reproduction number $\mathscr{R}_{0}$ can be determined using the method of next-generation matrix, as presented in [23, 24]. Thus, to determine $\mathscr{R}_{0}$, let us find the spectral radius of the matrix $F V^{-1}$, where

$$
\begin{aligned}
F & =\left(\begin{array}{cc}
0 & (1-p) \beta \frac{S^{*}}{K} \\
0 & 0
\end{array}\right), \\
V & =\left(\begin{array}{cc}
\tau+d+b & 0 \\
-(1-p) \phi & \mu_{B}-b_{B}
\end{array}\right) .
\end{aligned}
$$

Let $k_{1}=\tau+d+b$ and $k_{2}=\mu_{B}-b_{B}$, and we get the inverse of $V$ and $F V^{-1}$ as

$$
\begin{aligned}
V^{-1} & =\left(\begin{array}{cc}
\frac{1}{k_{1}} & 0 \\
\frac{(1-\theta) \phi}{k_{1} k_{2}} & \frac{1}{k_{2}}
\end{array}\right), \\
F V^{-1} & =\left(\begin{array}{cc}
\frac{(1-p)(1-\theta) \beta \phi S^{*}}{K k_{1} k_{2}} & (1-p) \beta \frac{S^{*}}{K k_{1}} \\
0 & 0
\end{array}\right) .
\end{aligned}
$$
be

Hence, the spectral radius of the matrix $F V^{-1}$ is found to

$$
\begin{aligned}
\mathscr{R}_{0} & =\frac{(1-p)(1-\theta) \beta \phi S^{*}}{K k_{1} k_{2}}, \\
& =\frac{(1-p) \beta b\left(b-\mu_{i}\right)}{\mu_{d}(\tau+d+b)(p \alpha+b)} \frac{(1-\theta) \phi}{K\left(\mu_{B}-b_{B}\right)} .
\end{aligned}
$$

The quantity $1 /(\tau+d+b)$ indicates the maximum time an individual is expected to stay in an infected cohort. The basic reproduction number consists of two terms which characterize the contribution from the different pathways to new infections with cholera. The quantity $\mathscr{R}_{0 h}=(((1-$ p) $\left.\left.\beta b\left(b-\mu_{i}\right)\right) /\left(\mu_{d}(\tau+d+b)(p \alpha+b)\right)\right)$ shows new infection of individuals due to person-to-person contact, and $\mathscr{R}_{0 B}=\left(((1-\theta) \phi) /\left(K\left(\mu_{B}-b_{B}\right)\right)\right)$ represents the new infection of individuals that results from consumption of the pathogen from the aquatic environment.

3.3. Stability of the Disease-Free Equilibrium Point. The dynamical system (1) is nonlinear. Its local stability is determined from the sign of the eigenvalues of the corresponding Jacobian matrix at the disease-free equilibrium point. The Jacobian matrix corresponding to the system in equation (1) at any point $E=(S, I, R, U, B)$ is 


$$
J(E)=\left(\begin{array}{ccccc}
-\left((1-p) \beta \frac{B}{K+B}+p \alpha+\mu\right) & 0 & (1-p) \rho & 0 & -(1-p) \beta \frac{K S}{(K+B)^{2}} \\
(1-p) \frac{\beta B}{K+B} & -(\tau+d+\mu) & 0 & 0 & (1-p) \beta \frac{K S}{(K+B)^{2}} \\
0 & -(\rho+\mu) & 0 & 0 \\
p \alpha & 0 & p \rho & -\mu & 0 \\
0 & (1-\theta) \phi & 0 & 0 & -\left(\mu_{B}-b_{B}\right)
\end{array}\right) .
$$

Therefore, the Jacobian matrix at the disease-free equilibrium point is

$$
J\left(E_{0}\right)=\left(\begin{array}{ccccc}
-(p \alpha+b) & 0 & (1-p) \rho & 0 & -\frac{(1-p) \beta S^{*}}{K} \\
0 & -(\tau+d+b) & 0 & 0 & \frac{(1-p) \beta S^{*}}{K} \\
0 & \tau & -(\rho+b) & 0 & 0 \\
p \alpha & 0 & p \rho & -b & 0 \\
0 & (1-\theta) \phi & 0 & 0 & -\left(\mu_{B}-b_{B}\right)
\end{array}\right) .
$$

Theorem 2 (local stability of the disease-free equilibrium point). If $\mathscr{R}_{0}<1$, then the disease-free equilibrium point $E_{0}$ of the dynamical system (1) is locally asymptotically stable, and if $\mathscr{R}_{0}>1$, then $E_{0}$ is unstable.

Proof. Consider that the characteristics equation of the Jacobian matrix at the disease-free equilibrium point is given by $\left|J\left(E_{0}\right)-\lambda I_{5}\right|=0$, where $I_{5}$ is the identity matrix and can be written as

$$
(\lambda+p \alpha+b)(\lambda+b)(\lambda+\rho+b)\left[\lambda^{2}+a_{1} \lambda+a_{2}\right]=0,
$$

where $a_{1}=k_{1}+k_{2}$ and $a_{2}=k_{1} k_{2}\left(1-\mathscr{R}_{0}\right)$.

The roots of the polynomial $(\lambda+p \alpha+b)(\lambda+b)(\lambda+\rho+$ $b)=0$ are a negative real value because $p \alpha+b>0, b>0$ and $\rho+b>0$. We use the Routh-Hurwitz criterion to show that the roots of the polynomial,

$$
\lambda^{2}+a_{1} \lambda+a_{2}=0
$$

have negative real values. To have negative real values for roots of the polynomial, in equation (19), both $a_{1}=k_{1}+k_{2}$ and $a_{2}=k_{1} k_{2}\left(1-\mathscr{R}_{0}\right)$ must be positive. $k_{1}=\tau+d+b>0$ and $k_{2}=\mu_{B}-b_{B}>0$ because all parameters are positive constants, and $\mu_{B}>b_{B}$. Thus, $a_{1}=k_{1}+k_{2}>0$ because it is the sum of the positive number and $a_{2}=k_{1} k_{2}\left(1-\mathscr{R}_{0}\right)>0$ if and only if $\mathscr{R}_{0}<1$. Hence, the roots of a polynomial in equation (19) have a negative real value. Therefore, the roots of the characteristic equation (18) have a negative real value which indicates the disease-free equilibrium point is locally asymptotically stable. This means, the disease cannot sustain in the population, and thus, it is eventually disappearing. On the other hand, if $a_{2}<0$, then $\mathscr{R}_{0}>1$. Thus, there exists at least one positive eigenvalue, and thus, the Routh-Hurwitz criterion tells us the disease-free equilibrium point is unstable. Biologically, the disease becomes endemic or the disease persists in the population.

Theorem 3 (global stability of the disease-free equilibrium point). If $\mathscr{R}_{0}<1$, then the cholera-free equilibrium point $E_{0}$ of the dynamical system in equation (1) is globally asymptotically stable, and if $\mathscr{R}_{0}>1$, then $E_{0}$ is unstable.

Proof. Consider a Lyapunov function $V(t)=B$. From the dynamical system in equation (1) by setting $(\mathrm{d} I / \mathrm{d} t)=0$, we get

$$
I=\frac{(1-p) \beta B S}{(\tau+d+\mu)(B+K)} .
$$


Moreover, $S=S^{*}, \mu=b$ at the disease-free equilibrium point, and $(B /(B+K)) \leq(B / K)$ and takes the equality. Then,

$$
\begin{aligned}
\frac{\mathrm{d} V}{\mathrm{~d} t} & =(1-\theta) \phi I+\left(b_{B}-\mu_{B}\right) B=\frac{(1-p)(1-\theta) \phi \beta S B}{(\tau+d+\mu)(B+K)}-\left(\mu_{B}-b_{B}\right) B \\
& \leq\left(\mu_{B}-b_{B}\right)\left(\frac{(1-p)(1-\theta) \phi \beta S^{*}}{K(\tau+d+b)\left(\mu_{B}-b_{B}\right)}-1\right) B, \quad \text { at } E_{0}, \\
& =\left(\mu_{B}-b_{B}\right)\left(\mathscr{R}_{0}-1\right) B .
\end{aligned}
$$

When $\mathscr{R}_{0} \leq 1, \mathrm{~d} V / \mathrm{d} t$ is negative semidefinite, with equality at $\mathscr{R}_{0}=1$. Therefore, the largest compact invariant set in $\Omega$ such that $(\mathrm{d} V / \mathrm{d} t)=0$ when $R_{0} \leq 1$ is the singleton $E_{0}$. Hence, by the LaSalle invariance principle [25], the disease-free equilibrium $E_{0}$ is globally asymptotically stable in $\Omega$ and unstable otherwise.

Epidemiologically, the implication of Theorem 3 is that when $\mathscr{R}_{0}$ is less than unity, a small inflow of cholera-infected individuals into the community does not yield an outbreak. The subsequent numbers of those infected will be less than that of their predecessors, and eventually, the disease will be eradicated. We note also that a number of parameters are necessary in reducing the disease threshold to a value $\mathscr{R}_{0}<1$.

3.4. Endemic Equilibrium Point. The endemic equilibrium points are steady-state solutions determined when $I>0$, and there exist at least one endemic equilibrium point, $E=(S, I, R, U, B)$.
Theorem 4 (existence of at least one endemic equilibrium point). If $I>0$, then $\mathscr{R}_{0}>1$ and there exists at least one endemic equilibrium point for the model in equation (1).

Proof. Suppose that $I>0$ and $d=0$ (there is no diseaseinduced death). Thus, we get $N=N^{*}=L$ and $\mu=b$. Setting $(\mathrm{d} B / \mathrm{d} t)=0$ and $(\mathrm{d} R / \mathrm{d} t)=0$, we obtain

$$
\begin{aligned}
& B=\frac{(1-\theta) \phi I}{k_{2}}, \\
& R=\frac{\tau I}{\rho+b} .
\end{aligned}
$$

Similarly, by setting $(\mathrm{d} S / \mathrm{d} t)+(\mathrm{d} I / \mathrm{d} t)+(\mathrm{d} R / \mathrm{d} t)=0$, we yield

$$
\begin{aligned}
& S=\frac{b N-((p \rho+b) R+b I)}{p \alpha+b}=S^{*}-\left(\frac{(p \rho+b) \tau+(b+\rho) b}{(b+\rho)(p \alpha+b)}\right) \\
& I \leq S^{*}=\frac{b N^{*}}{p \alpha+b} .
\end{aligned}
$$

Moreover, by setting $(\mathrm{d} U / \mathrm{d} t)=0$, we get

$$
\begin{aligned}
U= & \frac{p \alpha S+p \rho R}{b}=\frac{p \alpha}{b}\left(S^{*}-\left(\frac{(p \rho+b) \tau+(b+\rho) b}{(b+\rho)(p \alpha+b)}\right) I\right) \\
& +\frac{p \rho \tau I}{b(\rho+b)} .
\end{aligned}
$$

Finally, setting $(\mathrm{d} I / \mathrm{d} t)=0$ results in

$$
I=\frac{(1-p) \beta B S}{(B+K) k_{1}}=\frac{(1-p)(1-\theta) \beta \phi I\left(S^{*}-(((p \rho+b) \tau+(b+\rho) b) /((b+\rho)(p \alpha+b))) I\right)}{\left((1-\theta) \phi I+K k_{2}\right) k_{1}}
$$

and by solving I explicitly, we obtain

$$
\begin{aligned}
I & =\frac{K k_{1} k_{2}\left(\left((1-p)(1-\theta) \beta \phi S^{*}\right) /\left(K k_{1} k_{2}\right)-1\right)(b+\rho)(p \alpha+b)}{(1-\theta) \phi k_{1}(b+\rho)(p \alpha+b)+\pi}, \\
& =\frac{K k_{1} k_{2}\left(\mathscr{R}_{0}-1\right)(b+\rho)(p \alpha+b)}{(1-\theta) \phi k_{1}(b+\rho)(p \alpha+b)+\pi},
\end{aligned}
$$

where $\pi=(1-p)(1-\theta) \beta \phi((p \rho+b) \tau+(b+\rho) b)$. Here, we observe that $I>0$ if and only if $\mathscr{R}_{0}>1$. Therefore, if $I>0$, then $\mathscr{R}_{0}>1$ and there exists at least one endemic equilibrium point for the dynamical equation (1) given by

$$
\begin{aligned}
E & =\left(S_{*}, I_{*}, R_{*}, U_{*}, B_{*}\right), \text { where } \\
S_{*} & =S^{*}-\left(\frac{(p \rho+b) \tau+(b+\rho) b}{(b+\rho)(p \alpha+b)}\right) I_{*}, \\
I_{*} & =\frac{K k_{1} k_{2}\left(\mathscr{R}_{0}-1\right)(b+\rho)(p \alpha+b)}{(1-\theta) \phi k_{1}(b+\rho)(p \alpha+b)+\pi}, \\
R_{*} & =\frac{\tau I_{*}}{\rho+b}, \\
U_{*} & =\frac{p \alpha}{b}\left(S^{*}-\left(\frac{(p \rho+b) \tau+(b+\rho) b}{(b+\rho)(p \alpha+b)}\right) I_{*}\right)+\frac{p \rho \tau I_{*}}{b(\rho+b)}, \\
B_{*} & =\frac{(1-\theta) \phi I_{*}}{k_{2}} .
\end{aligned}
$$


Theorem 5 (global stability of the endemic equilibrium point). If $\mathscr{R}_{0}>1$, then the endemic equilibrium point $E$ of the dynamical system in equation (1) is globally asymptotically stable, and if $\mathscr{R}_{0}<1$, then $E$ is unstable.

$$
I=\frac{(1-p) \beta B S}{k_{1}(B+K)} .
$$

Proof. Consider a Lyapunov function $V(t)=B-B_{*}-$ $B_{*} \ln \left(B / B_{*}\right)$. From the dynamical system in equation (1), by setting $(\mathrm{d} I / \mathrm{d} t)=0$, we get

$$
\begin{aligned}
\frac{\mathrm{d} V}{\mathrm{~d} t} & =\frac{\mathrm{d} B}{\mathrm{~d} t}-\frac{B_{*}^{2}}{B} \frac{\mathrm{d} B}{\mathrm{~d} t}=(1-\theta) \phi I-k_{2} B-\frac{B_{*}^{2}}{B}\left[(1-\theta) \phi I-k_{2} B\right], \\
& =\frac{(1-p)(1-\theta) \phi \beta S B}{(B+K) k_{1}}-k_{2} B+\frac{B_{*}^{2}}{B}\left[k_{2} B-\frac{(1-p)(1-\theta) \phi \beta S B]}{(B+K) k_{1}}\right], \\
\text { let } \mu & =b \text { and } B=2 K \\
& =\frac{(1-p)(1-\theta) \phi \beta 2 K S}{3 K k_{1}}-2 k_{2} K+\frac{B_{*}^{2}}{2 K}\left[2 k_{2} K-\frac{(1-p)(1-\theta) \phi \beta 2 K S}{3 K k_{1}}\right] \\
& \leq \frac{(1-p)(1-\theta) \phi \beta 2 K S^{*}}{3 K k_{1}}-\frac{2 k_{2} K}{3}+\frac{B_{*}^{2}}{2 K}\left[2 k_{2} K-\frac{(1-p)(1-\theta) \phi \beta 2 K S^{*}}{K k_{1}}\right], \\
& =\frac{2}{3} k_{2} K\left(\mathscr{R}_{0}-1\right)+k_{2} B_{*}^{2}\left(1-\mathscr{R}_{0}\right),=k_{2}\left(1-\mathscr{R}_{0}\right)\left(-\frac{2}{3} K+B_{*}^{2}\right) \leq k_{2}\left(1-\mathscr{R}_{0}\right) B_{*}^{2} .
\end{aligned}
$$

When $\mathscr{R}_{0} \geq 1, \mathrm{~d} V / \mathrm{d} t$ is negative semidefinite, with equality at $\mathscr{R}_{0}=1$. Therefore, the largest invariant set in $(\mathrm{d} V / \mathrm{d} t)=0$ is the singleton $E$. By LaSalle's invariance principle [25], we therefore, conclude that the endemic equilibrium $E$ is globally asymptotically stable if $\mathscr{R}_{0}>1$.

\section{Bifurcation Analysis}

A bifurcation is a qualitative change of the phase portrait due to the variation of parameter values. The change in the qualitative character of a solution as a control parameter is varied is known as a bifurcation point. At the bifurcation point, the equilibrium points, or stability properties of the equilibrium points, or both, change. We investigate bifurcation analysis of the dynamical system (1) by using center manifold theory [26].

Theorem 6. If $a^{*}>a^{\circ}$, then the dynamical system equation (1) exhibits forward bifurcation at $R_{0}=1$, where $a^{*}=$ $\left(k_{1} /\left((p \alpha+b) S^{*}\right)\right)+k_{2} /((1-\theta) \phi K)$ and $a^{\circ}=(((1-p) \rho \tau) /$ $\left.\left((\rho+b)(p \alpha+b) S^{*}\right)\right)$.

Proof. We prove the possibility of bifurcation at $\mathscr{R}_{0}=1$ using the center manifold theorem [26]. For more convenience, let

$$
\begin{aligned}
S & =x_{1}, \\
I & =x_{2}, \\
R & =x_{3}, \\
U & =x_{4}, \\
B & =x_{5}, \\
\frac{\mathrm{d} S}{\mathrm{~d} t} & =f_{1}, \\
\frac{\mathrm{d} I}{\mathrm{~d} t} & =f_{2}, \\
\frac{\mathrm{d} R}{\mathrm{~d} t} & =f_{3}, \\
\frac{\mathrm{d} U}{\mathrm{~d} t} & =f_{4}, \\
\frac{\mathrm{d} B}{\mathrm{~d} t} & =f_{5} .
\end{aligned}
$$

We consider $\beta$ as the bifurcation parameter at $\mathscr{R}_{0}=1$, and we obtain $\beta=\left(\left(K k_{1} k_{2}\right) /\left((1-p)(1-\theta) \phi S^{*}\right)\right)$. The Jacobian matrix at disease-free equilibrium point $E_{0}$ given in equation (17) has a simple zero eigenvalue when $\beta=\left(\left(K k_{1} k_{2}\right) /\left((1-p)(1-\theta) \phi S^{*}\right)\right)$ or $\mathscr{R}_{0}=1$. 
The right eigenvector, $w=\left(w_{1}, w_{2}, w_{3}, w_{4}, w_{5}\right)^{t}$, associated with the simple zero eigenvalue of $J\left(E_{0}\right)$ when $\beta=$ $\left(\left(K k_{1} k_{2}\right) /\left((1-p)(1-\theta) \phi S^{*}\right)\right)$ can be obtained from $J\left(E_{0}\right) w=0$. The system of equation becomes

$$
\begin{array}{r}
(p \alpha+b) w_{1}+(1-p) \rho w_{3}-\frac{(1-p) \beta S^{*}}{K} w_{5}=0, \\
-k_{1} w_{2}+\frac{(1-p) \beta S^{*}}{K} w_{5}=0, \\
\tau w_{2}-(\rho+b) w_{3}=0, \\
p \alpha w_{1}+p \rho w_{3}-b w_{4}=0, \\
(1-\theta) \phi w_{2}-k_{2} w_{5}=0 .
\end{array}
$$

The solution of the system in (31) is obtained as

$$
\begin{aligned}
& w_{1}=\left(\frac{(1-p) \rho \tau}{(\rho+b)(p \alpha+b)}-\frac{k_{1}}{(p \alpha+b)}\right) w_{2}, \\
& w_{3}=\frac{\tau}{(\rho+b)} w_{2}, \\
& w_{4}=\frac{p \alpha}{b} w_{1}+\frac{p \rho}{b} w_{3}, \\
& w_{5}=\frac{(1-\theta) \phi}{k_{2}} w_{2} .
\end{aligned}
$$

In a similar manner, we compute the left eigenvector, $v=\left(v_{1}, v_{2}, v_{3}, v_{4}, v_{5}\right)$ associated with the simple zero eigenvalue of $J\left(E_{0}\right)$ when $\beta=\left(\left(K k_{1} k_{2}\right) /\left((1-p)(1-\theta) \phi S^{*}\right)\right)$ obtained from $v J\left(E_{0}\right)=0$, and its solution becomes

$$
\begin{aligned}
& v_{1}=v_{3}=v_{4}=0, \\
& v_{5}=\frac{k_{1}}{(1-\theta) \phi} v_{2},
\end{aligned}
$$

where $v_{2}$ is calculated to ensure that the eigenvectors satisfy the condition $v w=1$. Since $v_{1}=v_{3}=v_{4}=0$, we do not require the derivatives of $f_{1}, f_{3}$, and $f_{4}$. The only nonzero from the derivative of $f_{2}$ and $f_{5}$ is the following:

$$
\begin{aligned}
& \frac{\partial^{2} f_{2}\left(E_{0}\right)}{\partial x_{1} \partial x_{5}}=\frac{\partial^{2} f_{2}\left(E_{0}\right)}{\partial x_{5} \partial x_{1}}=\frac{(1-p) \beta}{K}, \\
& \frac{\partial^{2} f_{2}\left(E_{0}\right)}{\partial x_{2}^{2}}=-2 \frac{(1-p) \beta S^{*}}{K^{2}}, \\
& \frac{\partial^{2} f_{2}\left(E_{0}\right)}{\partial x_{5} \partial \beta}=\frac{(1-p) S^{*}}{K},
\end{aligned}
$$

and otherwise, all the other partial derivatives of $f_{2}$ and $f_{5}$ are zero. The direction of the bifurcation at $\mathscr{R}_{0}=1$ is determined from the signs of the bifurcation coefficients $a$ and $b$ given by

$$
\begin{aligned}
a & =\sum_{k, i, j=1}^{5} v_{k} w_{i} w_{j} \frac{\partial^{2} f_{k}\left(E_{0}\right)}{\partial x_{i} \partial x_{j}} \\
& =v_{2} w_{1} w_{5} \frac{(1-p) \beta}{K}+v_{2} w_{5} w_{1} \frac{(1-p) \beta}{K}-2 v_{2} w_{2} w_{2} \frac{(1-p) \beta S^{*}}{K^{2}} \\
& =2 v_{2} w_{1} w_{5} \frac{(1-p) \beta}{K}-2 v_{2} w_{2}^{2} \frac{(1-p) \beta S^{*}}{K^{2}} \\
& =-2 v_{2} w_{2}^{2} k_{1}\left(\frac{k_{1}}{(p \alpha+b) S^{*}}+\frac{k_{2}}{(1-\theta) \phi K}-\frac{(1-p) \rho \tau}{(\rho+b)(p \alpha+b) S^{*}}\right), \\
& =-2 v_{2} w_{2}^{2} k_{2}\left(a^{*}-a^{\circ}\right), \\
b & =\sum_{k, i=1}^{5} v_{k} w_{i} \frac{\partial^{2} f_{k}(0,0)}{\partial x_{i} \partial \beta}=v_{2} w_{5} \frac{(1-p) S^{*}}{K} \\
& =\frac{v_{2} w_{2}(1-p)(1-\theta) \phi S^{*}}{k_{2} K}>0 .
\end{aligned}
$$

If $a^{*}>a^{\circ}$, then $a<0$. We observe that the coefficient $b$ is always positive and the sign of the coefficient $a$ is negative provided that $a^{*}>a^{\circ}$. Therefore, the dynamical system in equation (1) exhibits a forward bifurcation using the center manifold theorem [26]. This indicates that an exchange of stability occurs at $\mathscr{R}_{0}=1$. Using expression for $I_{*}$ in the endemic equilibrium and parameter values in Table 2, we plotted a forward bifurcation diagram as shown in Figure 2.

\section{Sensitivity Analysis of Parameters}

In epidemiology, it is crucial to understand the relative importance of the different factors responsible for its transmission and prevalence in determining how to best reduce mortality and morbidity due to the disease. Sensitivity analysis is used to study the sensitivity changes of parameters on the transmission and prevalence of the disease $[27,28]$. Sensitivity indices are used to measure the relative change in a state variable as a parameter value changes. The normalized forward sensitivity index of a variable to a parameter is determined by the ratio of the relative change in the variable to the relative change in the parameter. If the variable is a differentiable function of the parameter, then the sensitivity index can be defined using partial derivatives as follows: the normalized forward sensitivity index of a variable, $\eta$, that depends differentiably on a parameter, $\xi$, is defined as

$$
\Lambda_{\xi}^{\eta}=\frac{\partial \eta}{\partial \xi} \times \frac{\xi}{\eta}
$$

where $\xi$ represents all the basic parameters $[27,28]$.

$$
\begin{gathered}
\text { For } \quad \text { example, } \\
\begin{array}{c}
\left.\left.(1-\theta) \beta \phi b\left(b-\mu_{i}\right)\right) /\left(K \mu_{d}(\tau+d+b)\left(\mu_{B}-b_{B}\right)(p \alpha+b)\right)\right), \\
\Lambda_{p}^{\mathscr{R}_{0}}=\frac{\partial \mathscr{R}_{0}}{\partial p} \times \frac{p}{\mathscr{R}_{0}}=-\frac{p(\alpha+b)}{(1-p)(p \alpha+b)} .
\end{array}
\end{gathered}
$$


TABLe 2: Parameter values used for sensitivity analysis and simulation.

\begin{tabular}{lccc}
\hline Parameter & Value & Sources & Sensitivity indices \\
\hline$b$ & 0.00082 & Assumed & 2.03 \\
$\mu_{d}$ & $3.245 \times 10^{-8}$ & Assumed & -1 \\
$\beta$ & 1 & {$[5]$} & 1 \\
$\tau$ & 0.2 & {$[16]$} & -0.95 \\
$\rho$ & 0.01 & Assumed & 0 \\
$K$ & $10^{6}$ & {$[5]$} & -1 \\
$\theta$ & 0.8 & Assumed & -4 \\
$\mu_{i}$ & $4.21 \times 10^{-5}$ & Assumed & -0.05 \\
$d$ & 0.01 & {$[15]$} & -0.05 \\
$b_{B}-\mu_{B}$ & -0.33 & {$[5]$} & -1 \\
$\alpha$ & 0.1 & Assumed & -0.98 \\
$\phi$ & 10 & {$[16]$} & 1 \\
$p$ & 0.7 & Assumed & -3.32 \\
\hline
\end{tabular}

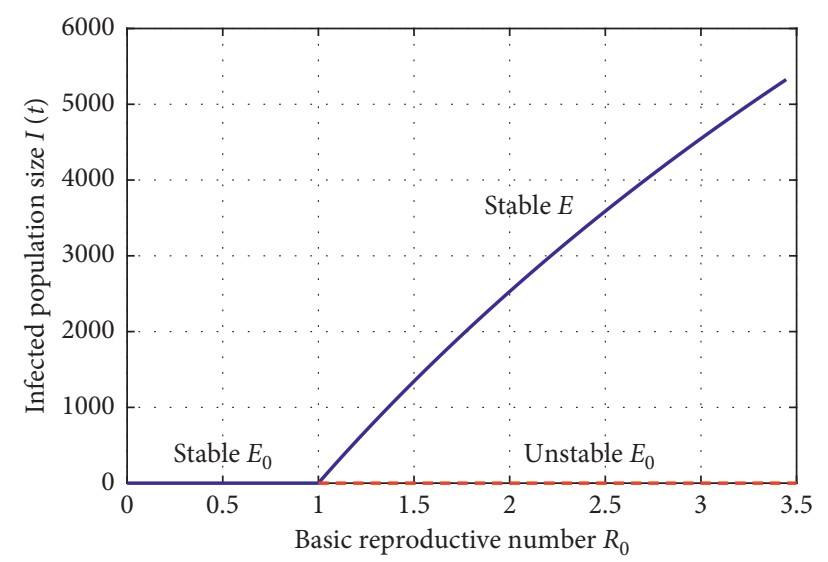

FIgURE 2: Forward bifurcation of the dynamical system in equation (1) at $\mathscr{R}_{0}=1$.

The sensitivity indices of the basic reproductive number with respect to parameters are found in Table 2. Thus, the negative indices are $\left(p, \theta, K, \mu_{d}, \mu_{B}-b_{B}, \tau, \alpha\right)$ indicating that they had a negative impact on the transmission of the disease as the values of the parameter are increasing. The positive indices are $(b, \beta, \phi)$ indicating that they had a great impact on the transmission of the disease as the values of the parameter are increasing. This indicates that, to eradicate cholera, stakeholders should devise strategies and implement it to reduce these parameters.

\section{Numerical Simulation}

In this section, numerical simulations for model (1) is carried out. The parameter values that are used for numerical simulations are listed in Table 2 in which the parameter values of $p$ and $\theta$ are varied. It is verified that the disease-free equilibrium point is stable when $\mathscr{R}_{0}<1$ and unstable whenever $\mathscr{R}_{0}>1$.

Figures 3-6 are the time series solution of individual cohorts and the concentration of the cholera bacterium in an aquatic reservoir when $\mathscr{R}_{0}=0.0024<1, \mathscr{R}_{0}=0.0120<1$, $\mathscr{R}_{0}=0.6891<1$, and $\mathscr{R}_{0}=3.4457>1$. The total individuals and protected individuals are coincided and increased towards the carrying capacity, whereas the susceptible, infected, and recovered individuals and the concentration of cholera bacterium decreased rapidly and became zero, see Figures 3 and 4 . The parameter values for the plot of Figure 3 are in Table 2, and the parameter values for the plot of Figure 4 are in Table 2 by replacing $\theta=0$. This indicates that the disease dies out when the progression rate of susceptible and recovered individuals to the protected class due to the compliance of hygienic, ingestion of cholera bacterium and contact with cholera patients, is increased more than the recruitment rate (i.e., $p \alpha>b)$. Moreover, the total individuals and susceptible individuals attract towards the carrying capacity, whereas the protected, infected, recovered individuals and the concentration of cholera bacterium decreased and attracted towards zero for the parameter value in Table 2 and $p=0$ or for $\mathscr{R}_{0}=0.6891<1$, see Figure 5 . This indicates that the disease also dies out when infected individuals comply to their sanitation. In other words, the disease is not transmitted if the infected individuals do not contribute the bacterium to the aquatic reservoir due to keeping sanitation of an infected individual. This indicates that reducing the contribution of the concentrated bacteria by infected individuals reduces the disease. 


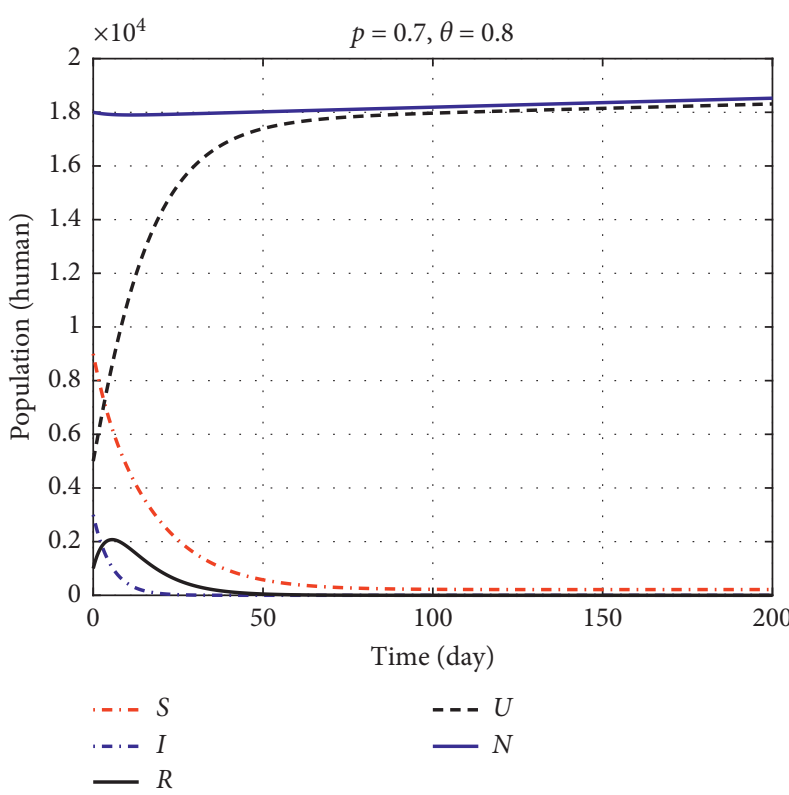

(a)

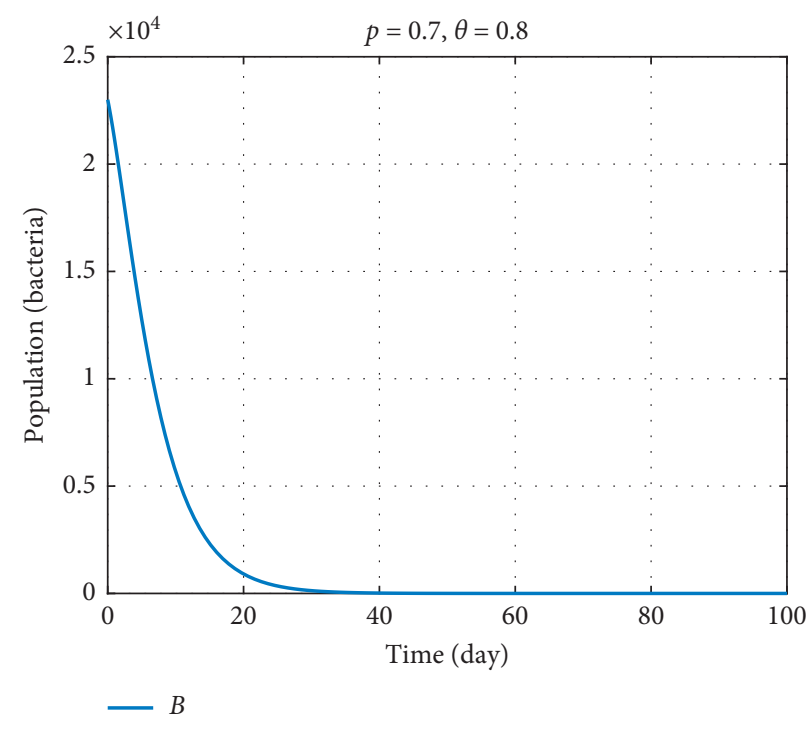

(b)

Figure 3: Time series solution of individual (a) and bacteria (b) cohorts when $p=0.7$ and $\theta=0.8$. The diseases die out $\left(\mathscr{R}_{0}=0.0024<1\right)$.

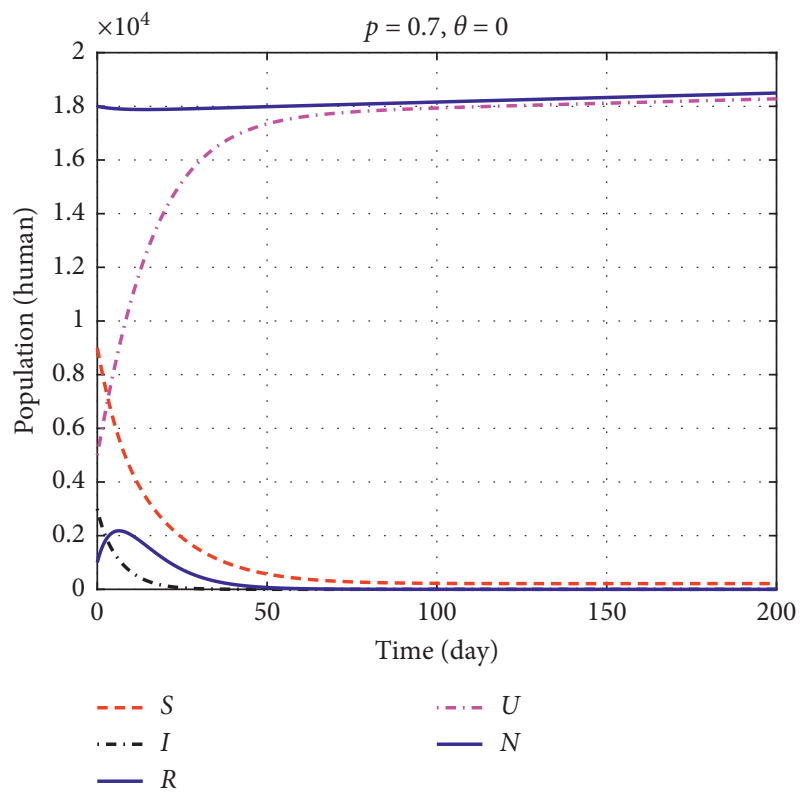

(a)

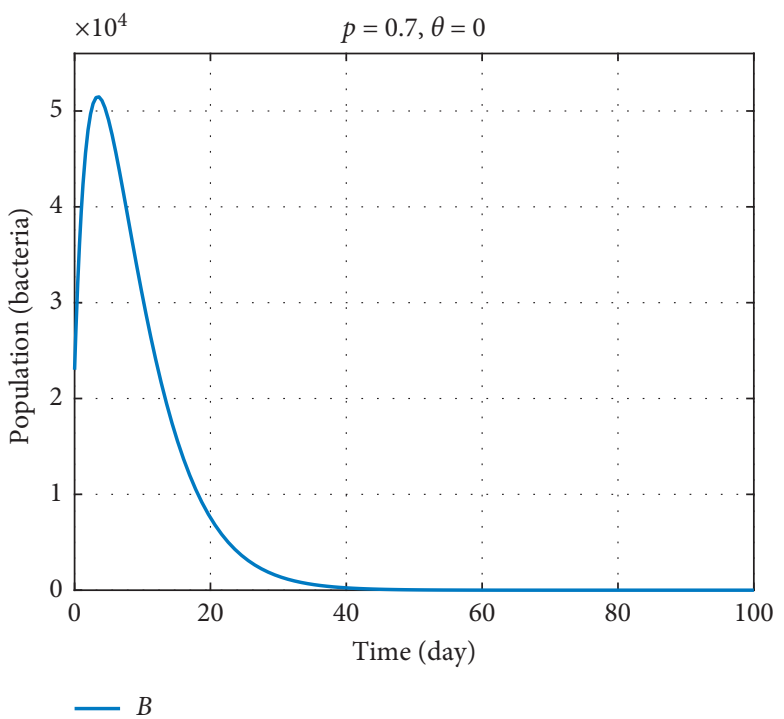

(b)

Figure 4: Time series solution of individual (a) and bacteria (b) cohorts when $p=0.7$ and $\theta=0$. The diseases die out $\left(\mathscr{R}_{0}=0.0120<1\right)$.

The solution curves do not settle down to their corresponding disease-free equilibrium point in the absence of implementation of adequate prevention measures, see Figure 6. That is, for parameter values in Table 2 with $p=\theta=0$ or $\mathscr{R}_{0}=3.4457>1$, the susceptible, protected, and total individuals decreased. The infected, recovered, and the concentration of cholera bacterium are nonzero after initially increasing and decreasing. This indicates that the disease infects and kills many people in the absence of knowledge and compliance of the prevention measure properly.

In summary, having knowledge and compliance of the prevention measure properly reduces the basic reproductive 


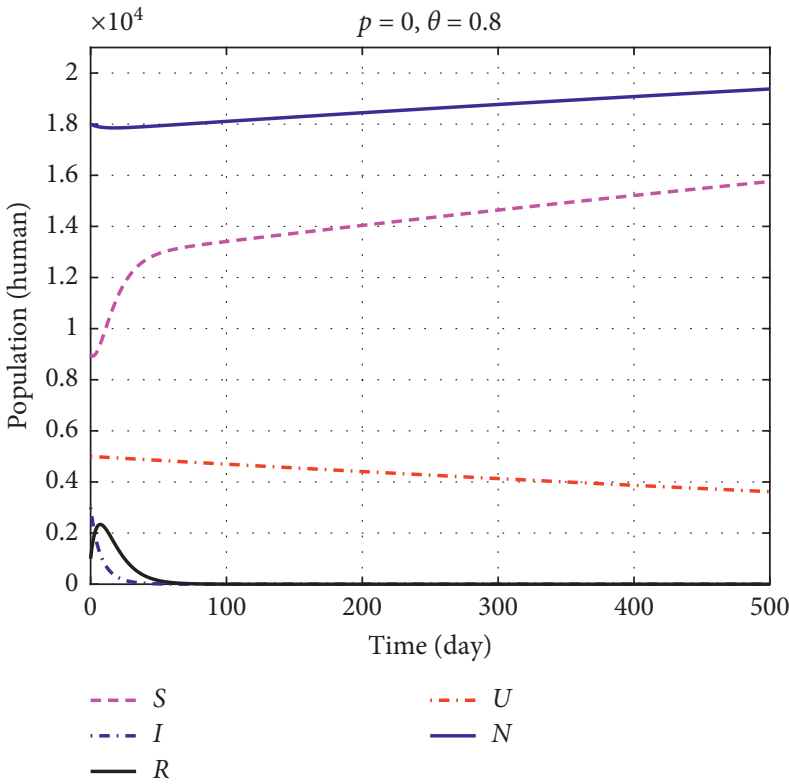

(a)

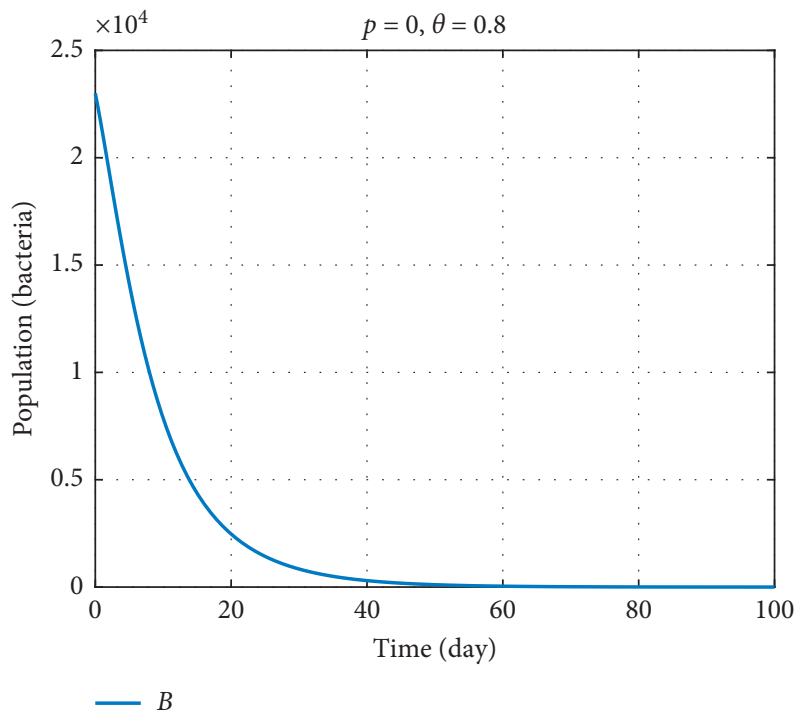

(b)

Figure 5: Time series solution of individual (a) and bacteria (b) cohorts when $p=0$ and $\theta=0.8$. The diseases die out $\left(\mathscr{R}_{0}=0.6891<1\right)$.

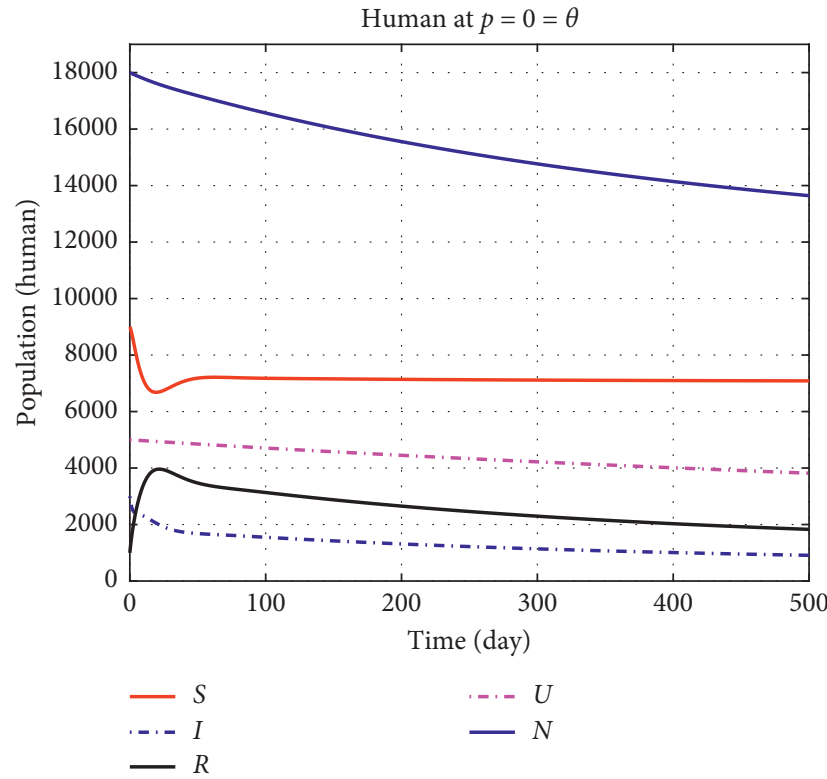

(a)

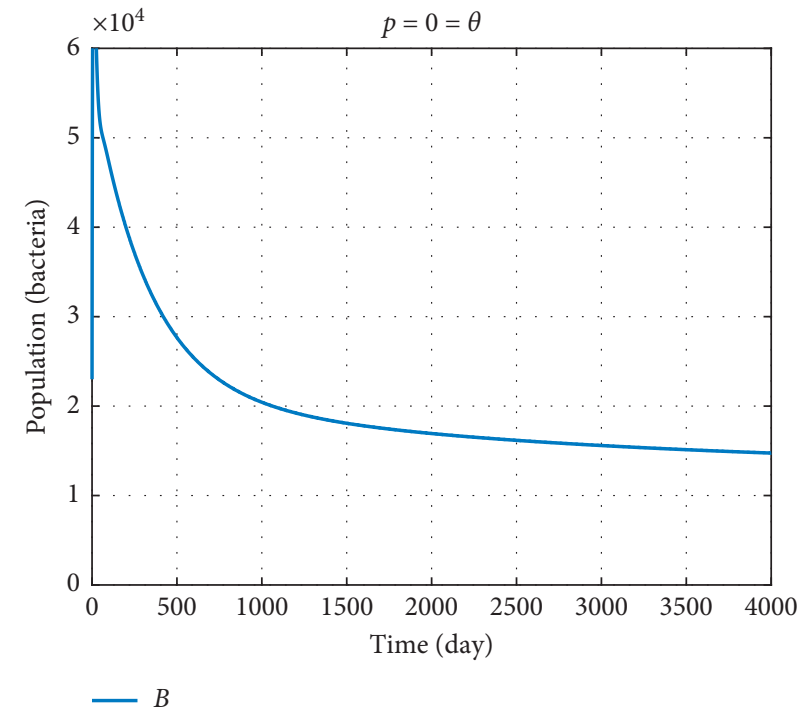

(b)

Figure 6: Time series solution of individual (a) and bacteria (b) cohorts when $p=\theta=0$. The diseases do not die out $\left(\mathscr{R}_{0}=3.4457>1\right)$.

number less than one. This means that each infected individual over infectious time transmits the infection on average to less than one new individual so that the disease dies out. When an individual has no knowledge and compliance of the prevention measures properly, the basic reproductive number increases greater than one. This means that each infected individual over an infection period transmits the infection on average to more than one new individual so that the disease spreads in the population.

\section{Conclusions}

In this study, effects of implementation of adequate preventive measures on the dynamics of cholera are examined using a mathematical model. To analyze the effect of implementation of preventive measures, a deterministic mathematical model system of nonlinear ordinary differential equations with the inclusion of the protected individuals cohort as one cohort is formulated. The disease-free 
equilibrium point and basic reproductive number are determined. The disease-free equilibrium point is locally and globally asymptotically stable when $\mathscr{R}_{0}<1$ and otherwise, unstable. The model is found to show the phenomenon of forward bifurcation at $\mathscr{R}_{0}=1$ which indicates that a stable disease-free equilibrium does not coexist with a stable endemic equilibrium. The numerical result shows $\mathscr{R}_{0}<1$ when susceptible and recovered individual compliance of hygienic, ingestion of cholera bacterium and contact with cholera patients, is increased or the compliance of sanitation of infected people is increased. Thus, having knowledge and compliance of the prevention measures properly reduces the basic reproductive number below one. On the contrary, when an individual has no knowledge and compliance of the prevention measure properly, the basic reproductive number increases greater than one. The fraction of the compliance of hygiene, ingestion of cholera bacterium and contact with cholera patients of the susceptible and recovered cohorts $(p)$, and the fraction of the compliance of sanitation of the infected cohort $(\theta)$ are the most sensitive parameters that can be used to control the propagation of the disease. Hence, these parameters are those that should be targeted the most by stakeholders to combat the disease. Therefore, from the qualitative study, it is concluded that the disease can be controlled and eliminated from the community if susceptible and recovered individuals comply with hygiene, ingestion of cholera bacterium and contact with cholera patients, or infected individuals comply with sanitation.

\section{Data Availability}

The parameter values and initial values to support this study are from the literature and assumptions.

\section{Conflicts of Interest}

The authors have no conflicts of interest to declare.

\section{References}

[1] Cholera-World Health Organization, 2019, https://www. who.int/news-room/fact-sheets/detail/cholera.

[2] World Health Organization, Vaccine-Preventable Diseases Surveillance Standards, World Health Organization, Geneva, Switzerland, 2018, https:/www.who.int/immunization/monitoring surveillance/burden/vpd/WHO_SurveillanceVaccinePreventable_ 02_Cholera_R2.pdf.

[3] P. Panja, "Optimal control analysis of a cholera epidemic model," Biophysical Reviews and Letters, vol. 14, no. 1, pp. 27-48, 2019.

[4] J. Lilje, H.-J. Mosler, and H. Kessely, "Factors determining water treatment behavior for the prevention of cholera in Chad," The American Journal of Tropical Medicine and Hygiene, vol. 93, no. 1, pp. 57-65, 2015.

[5] C. T. Codeco, "Endemic and epidemic dynamics of cholera: the role of the aquatic reservoir," BMC Infectious Diseases, vol. 1, no. 1, 2001.

[6] E. Bertuzzo, S. Azaele, A. Maritan, M. Gatto, I. RodriguezIturbe, and A. Rinaldo, "On the space-time evolution of a cholera epidemic," Water Resources Research, vol. 44, no. 1, 2008.

[7] E. Bertuzzo, R. Casagrandi, M. Gatto, I. Rodriguez-Iturbe, and A. Rinaldo, "On spatially explicit models of cholera epidemics," Journal of the Royal Society Interface, vol. 7, no. 43, pp. 321-333, 2010.

[8] L. Mari, E. Bertuzzo, L. Righetto et al., "Modelling cholera epidemics: the role of waterways, human mobility and sanitation," Journal of the Royal Society Interface, vol. 9, no. 67, pp. 376-388, 2012.

[9] A. A. Ayoade, M. O. Ibrahim, O. J. Peter, and F. A. Oguntolu, "On the global stability of cholera model with prevention and control," Malaysian Journal of Computing, vol. 3, no. 1, pp. 28-36, 2018.

[10] C. Ratchford and J. Wang, "Modeling cholera dynamics at multiple scales: environmental evolution, between-host transmission, and within-host interaction," Mathematical Biosciences and Engineering, vol. 16, no. 2, pp. 782-812, 2019.

[11] F. Nyabadza, J. M. Aduamah, and J. Mushanyu, "Modelling cholera transmission dynamics in the presence of limited resources," BMC Research Notes, vol. 12, no. 1, p. 475, 2019.

[12] J. P. Tian and J. Wang, "Global stability for cholera epidemic models," Mathematical Biosciences, vol. 232, no. 1, pp. 31-41, 2011.

[13] J. Wang and C. Modnak, "Modeling cholera dynamic with control," Canadian Applied Mathematics Quarterly, vol. 19, no. 3, pp. 255-273, 2011.

[14] K. A. Eustace, S. Osman, and M. Wainaina, "Mathematical modelling and analysis of the dynamics of cholera," Global Journal of Pure and Applied Mathematics, vol. 14, no. 9, pp. 1259-1275, 2018.

[15] N. Kwasi-Do Ohene Opoku and C. Afriyiel, "The role of control measures and the environment in the transmission dynamics of cholera," Abstract and Applied Analysis, vol. 2020, Article ID 2485979, 16 pages, 2020.

[16] S. Liao and W. Yang, "On the dynamic of a vaccination model with multiple transmission ways," International Journal of Applied Mathematics and Computer Science, vol. 23, no. 4, pp. 761-772, 2013.

[17] S. Fatima, I. Krishnarajah, M. Z. A. M. Jaffar, and M. B. Adam, "A mathematical model for the control of cholera in Nigeria," Research Journal of Environmental and Earth Sciences, vol. 6, no. 6, pp. 321-325, 2014.

[18] T. Bakhtiar, "Optimal intervention strategies for cholera outbreak by education and chlorination," Earth and Environmental Science, vol. 31, no. 1, 2016.

[19] Y. Cheng, J. Wang, and X. Yang, "On the global stability of a generalized cholera epidemiological model," Journal of Biological Dynamics, vol. 6, no. 2, pp. 1088-1104, 2012.

[20] A. Kumar, M. Kumar, and Nilam, "A study on the stability behavior of an epidemic model with ratio-dependent incidence and saturated treatment," Theory in Biosciences, vol. 139, no. 2, pp. 225-234, 2020.

[21] M. Al-Adydah, A. Mwasa, J. M. Tchuenche, and R. J. Smith, "Modeling cholera disease with education and chlorination," Journal of Biological Systems, vol. 21, no. 4, 2013.

[22] V. D. Nguyen, F. Dafae, C. Bopp et al., "Cholera epidemic associated with consumption of unsafe drinking water and street-vended water-eastern freetown, Sierra Leone, 2012," The American Journal of Tropical Medicine and Hygiene, vol. 90, no. 3, pp. 518-523, 2014.

[23] H. M. Yang, "The basic reproduction number obtained from Jacobian and next generation matrices-a case study of 
dengue transmission modelling," BioSystems, vol. 126, pp. 52-75, 2014.

[24] O. Diekmann, J. A. P. Heesterbeek, and M. G. Roberts, “The construction of next-generation matrices for compartmental epidemic models," Journal of the Royal Society Interface, vol. 7, no. 47, pp. 873-885, 2010.

[25] J. P. LaSalle, "The stability of dynamical systems," Society for Industrial and Applied Mathematics, vol. 21, no. 3, pp. 418420, 1979.

[26] C. Castillo-Chavez and B. Song, "Dynamical models of tuberculosis and their applications," Mathematical Biosciences and Engineering, vol. 1, no. 2, pp. 361-404, 2004.

[27] H. T. Alemneh, O. D. Makinde, and D. M. Theuri, "Ecoepidemiological model and analysis of MSV disease transmission dynamics in maize plant," International Journal of Mathematics and Mathematical Sciences, vol. 2019, Article ID 7965232, 14 pages, 2019.

[28] N. Chitnis, J. M. Hyman, and J. M. Cushing, "Determining important parameters in the spread of malaria through the sensitivity analysis of a mathematical model," Bulletin of Mathematical Biology, vol. 70, no. 5, pp. 1272-1296, 2008. 